\title{
Toward understanding the molecular mechanisms governing plant hormone actions: A brief introduction to the Major Research Program "Molecular mechanisms of plant hormone actions" funded by the National Natural Science Foundation of China (NSFC)
}

\author{
LI ChuanYou \& LI JiaYang
}

State Key Laboratory of Plant Genomics, National Centre for Plant Gene Research, Institute of Genetics and Developmental Biology, Chinese Academy of Sciences, Beijing 100101, China

Decades of physiological and molecular studies have demonstrated that plants rely on a diverse set of small molecule hormones to regulate every aspect of their biological processes including development, growth and adaptation. The way in which plant hormones influence plant biology depends on: (1) the amount of hormones present: this is regulated by biosynthesis, degradation, modification/conjugation, and transport/movement-mediated distribution; (2) the sensitivity of the tissue: this is regulated by the perception and the subsequent signal transduction event; (3) hormone cross-talk. The publication of the whole genome sequences of the model systems of Arabidopsis and rice, together with the advent of multidisciplinary approaches, opened the door to successful experimentation on plant hormone actions. A long-term goal of this project is to address, at the molecular level, key questions concerning plant hormone actions. This will be done in the reference species of Arabidopsis and rice using the tools and philosophy of systems biology. These studies are also expected to provide a new outlook upon how to value and utilize the "built-in" potentials of plants for the improvement of crops in an environmentally friendly and sustainable manner.

In order to realize the scientific goals of this research plan, the following scientific issues will be addressed:

(i) The molecular basis of hormone biosynthesis, metabolism, and its regulation; (ii) Hormone perception and signal transduction;

(iii) Signal cross-talks in hormone actions;

(iv) The functioning of hormones in organ development and plant growth;

(v) Hormonal regulation of plant responses to biotic and abiotic stresses;

(vi) Hormone analysis. The development of sensitive and accurate analytical methods for determining the hormone levels in plant tissues is essential for elucidating the role and function of plant hormones. This project encourages the development of a user-friendly bench-top mass spectrometersbased hormone measurement platform, as well as novel hormone analytical techniques at the tissue and cellular levels.

The Steering Committee of the Research Program, which consists of 7 experts in the fields of plant biology and chemistry, will function as a virtual 'umbrella organization' for the project by coordinating and facilitating research along the vision proposed in this research program. The International Consultation Committee will provide scientific guidance to this research program. Individual investigators are encouraged to conduct creative and innovative research which will benefit the overall goals of the project. Proposals will be peer reviewed through the ordinary NSFC channel. Once granted, the PI must submit a project report to the NSFC annually, and the Steering Committee is responsible for evaluation of the research progress. 